\title{
Temporomandibular Joint Remodeling for the Treatment of Temporomandibular Joint Disorders - A Clinical Case Study
}

\author{
Rosita Wurgaft $^{1}$ and Ricky W.K. Wong $^{*}, 2$ \\ ${ }^{1}$ University of Chile, Chile \\ ${ }^{2}$ University of Hong Kong, Hong Kong
}

\begin{abstract}
Introduction: The author (R. Wurgaft) has tried a unique way of splint therapy in the treatment of temporomandibular disorder. An orthopedic splint that only contact in the posterior occlusion, actually only the most distal contacts, is designed to distalize the mandible, and in turn to produce tissue remodeling of the temporomandibular joint. With this technique, it is possible to treat temporomandibular disorders in both adults and children by producing tissue remodeling. Method: Two clinical cases using this technique were performed. Result: improvement in temporomandibular disorder was obtained. Conclusion: It is possible for orthopedic splint with medial and posterior contacts only to produce TMJ remodeling in young and adult patients and to improve the TMD. Further clinical studies are warranted.
\end{abstract}

\section{INTRODUCTION}

Temporomandibular disorders (TMDs) represent a group of painful conditions involving the muscles of mastication and the temporomandibular joint (TMJ) that frequently encountered in general clinical practice, with a prevalence in the general population up to $12 \%$ [1-3]. Pain can be present at any stage of TMD and is a significant part of the symptoms that prompt patients to seek treatment [4].

Splint therapy is considered to be a major treatment option of TMD. There is strong evidence that occlusal splint therapy is successful in the treatment of TMD but there is no consensus of opinion on how splints work [5]. McNeill [6] pointed out several possible functions of splint therapy in the treatment of TMD: Alter occlusal forces; prevent wear and mobility of teeth; reduce bruxism and parafunction; treatment masticatory muscle pain and dysfunction and alter the structural relationships of the TMJ.

One of the most commonly used splint for the treatment of TMD is stabilization splints (Tanner appliance, Fox appliance, Michigan splint, centric relation (CR) appliance. It provides a temporary and removable ideal occlusion (centric occlusion equals to $\mathrm{CR}$, anterior guidance, no posterior interferences). It is usually made on the arch with the most missing teeth; with full occlusal coverage; with simultaneous and even contact between the splint and all opposing teeth in the CR position. A balanced occlusion should be provided between the splint and one cusp tip of every opposing tooth from the canines distally [5].

However, the stabilization splint is not always successful. To find a possible alternative to the stabilization splint, the author ( $\mathrm{R}$ Wurgaft) has tried a unique way of splint therapy in the treatment of TMD. The aim, unlike stabilization splint, is not to provide a removable ideal occlusion but to distalize

*Address correspondence to these authors at the $2 / \mathrm{F}$, Orthodontics, Prince Philip Dental Hospital, 34 Hospital Road, Sai Ying Pun, Hong Kong; Tel: 852-28590554; Fax: 852-25593803; E-mail: fyoung@hkucc.hku.hk the mandible and to produce tissue remodeling. It is an orthopedic splint that only allows contact in the posterior occlusion, actually only the most distal contacts; it is designed to distalize the mandible, and in turn to produce tissue remodeling of the TMJ. With this technique, the author is able to treat TMDs in both adults and children by producing tissue remodeling. The research question is that a splint that distalizes the mandible may also produce TMJ remodeling and improve TMD. In this preliminary study, two clinical cases using this technique were performed.

\section{SUBJECTS, METHODS AND RESULTS}

\section{Case 1: Male 12 Years Old (Fig. 1a-e)}

The patient is skeletal class III malocclusion with slight mandibular deviation to the right side. During mouth opening the mandible deviate to the left side. He complained of difficulty and pain with mouth opening. There was headache; bad sleep quality and attention deficiency during school classes. Examination also showed neck stiffness and sensibility.

Intraoral examination showed that the occlusal trauma was bigger in the left side, because it had more occlusal contacts in this side. It was possible this produced the morphofunctional asymmetry of both condyles and mandibular rami.

For his TMD treatment, an orthopedic splint that only contacted in the posterior sectors, actually only the most distal contact was used to distalize the mandible.

This mandibular posterior movement is possible because of the tissue remodeling of the joint. This is produced by changing the function and the forces that the tissues receive towards the extra cellular matrix.

The treatment length was 4 months. At the beginning, the occlusal contact was higher in the right side. This was to equilibrate the function that was overloaded in the left side and to induce symmetric growth of the condyles. 
The higher contact in the right side was left until there was no pain during mouth opening; no click and no mandibular deviation were observed.

With this method, the patient was seen once a week in the first stage of treatment until the pain disappeared or symmetrical function was obtained. Usually the splint was only used at night.

The stage 2 radiographs and pictures (Fig. 1f-j) showed that there were important orthopedic changes occurred in the condylar region. At this stage there were improvements in all

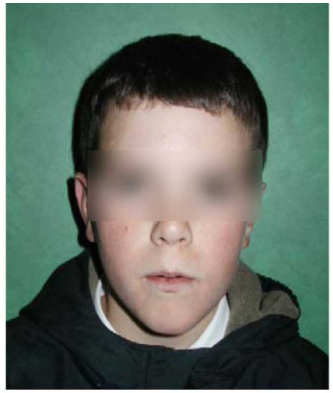

a

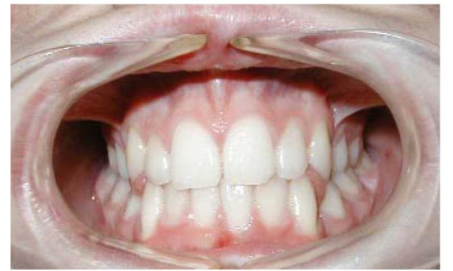

b

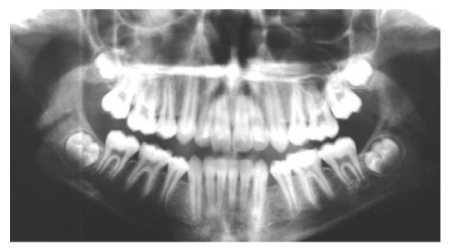

c

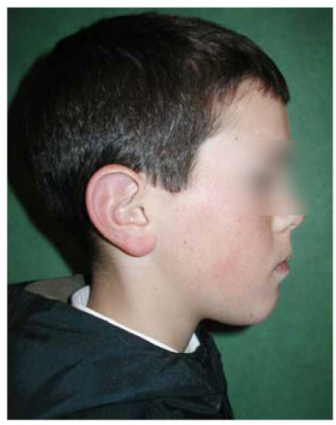

d

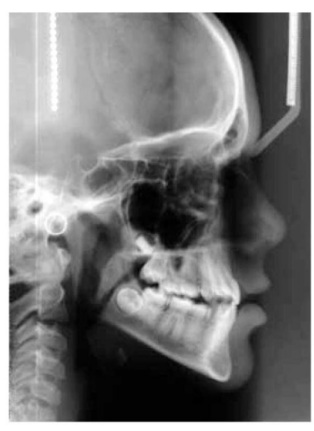

e
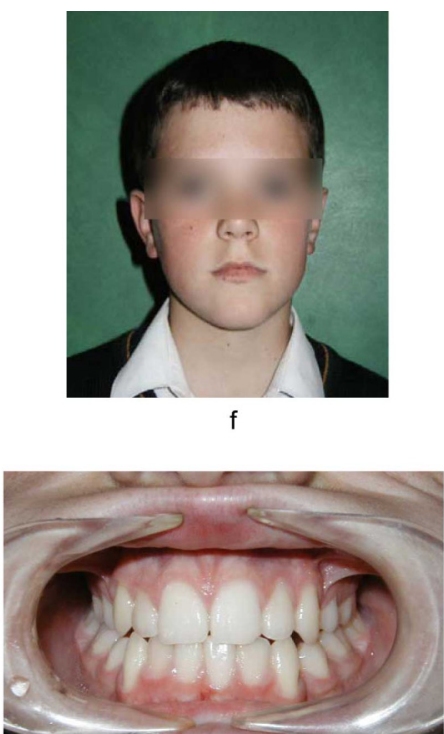

g

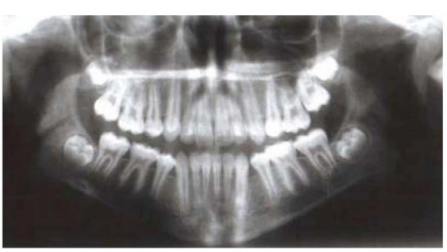

h

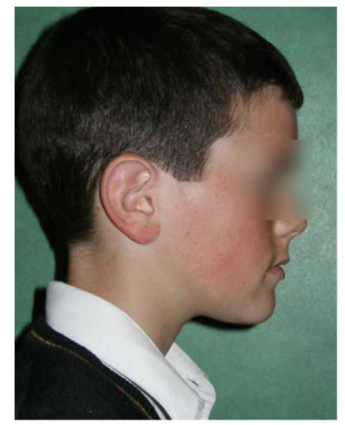

i

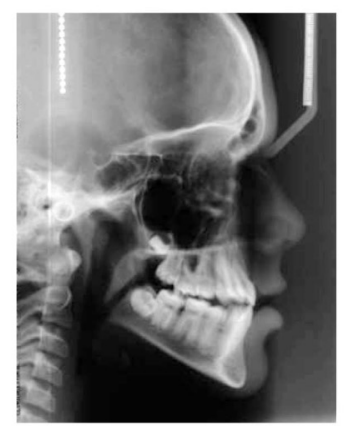

j
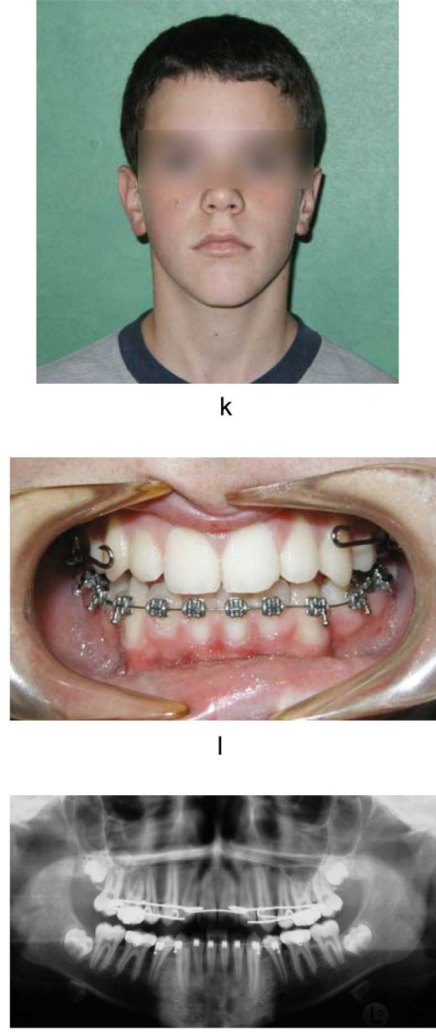

m

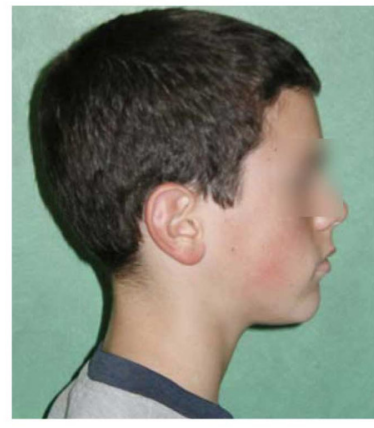

n

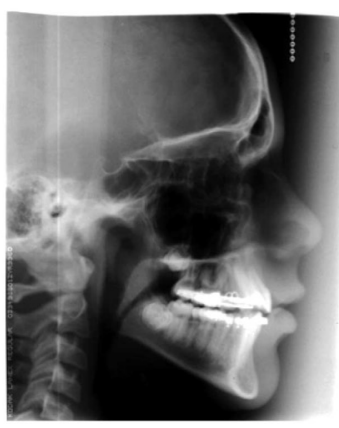

0

Fig. (1). Case 1. 
the TMD symptoms described previously. This patient could be treated by the orthodontics without surgery. In the stage 3 (Fig. 1, k-o) he was under orthodontic treatment. Rickett's superimpositions of pre- and postoperative radiographs showed that there was clockwise rotation of the occlusion plane, resulting an opening of facial axis and an improvement of the facial profile (Fig. 2). The details of the orthodontic treatment were not included as this was not the primary aim of this article.

In the experience of the author, with this kind of splints (Fig. 3, note only posterior contacts) tissues change in the TMJ in all patients of the author were obtained irrespective to the age of the patients. It is possible the speed and the amount of change are different, but there is still TMJ remodeling, as can be seen in the following adult case. Whether this is true in general requires further studies.

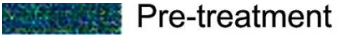

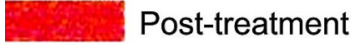

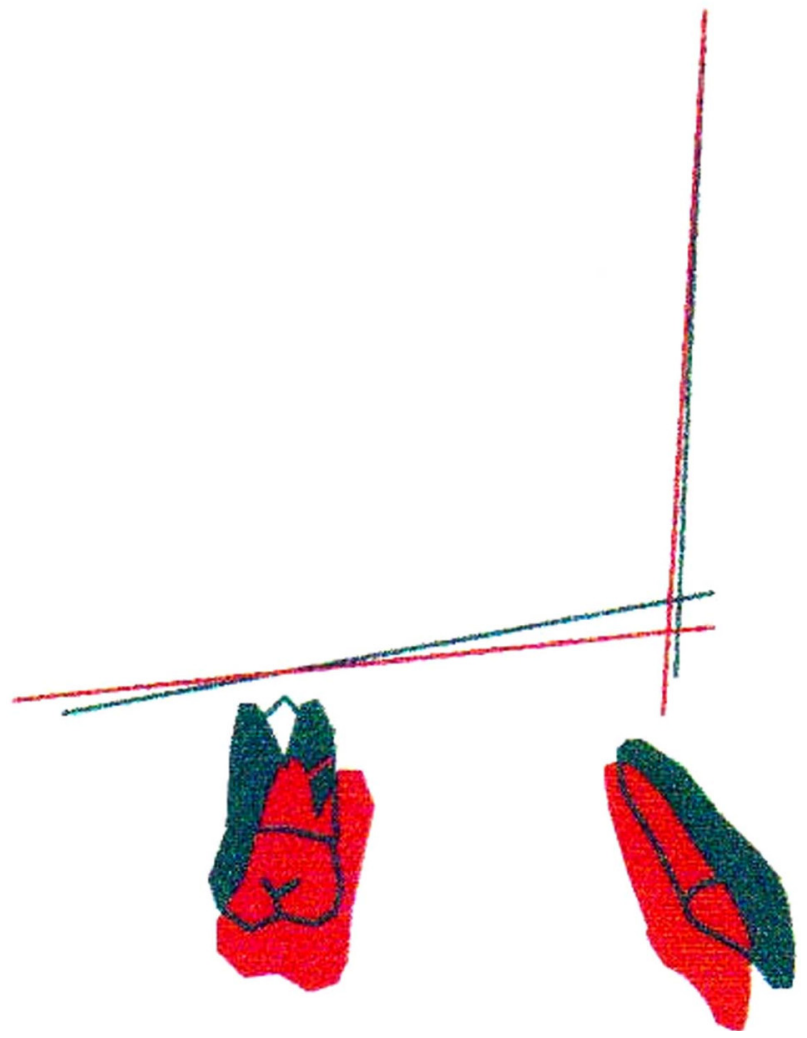

Fig. (2a). Rickett's superimposition along Nasion Basion line at Nasion. Pretreatment in blue and posttreatment in red. There was clockwise rotation of the occlusion plane.

\section{Case 2: Female 22 Years Old (Figs. 4-6).}

This patient was presented with TMD with pain of right TMJ and difficulty and pain during mouth opening. She also had headaches, ear pain, and pain at shoulder and right arm. She puts her tongue in the anterior tooth arch zone. Extraorally she presented with facial and neck muscular asymmetry. Examination showed neck and right trapezium stiffness and sensibility. Intraorally there was anterior open bite and she had occlusal contacts just in the second molars. The

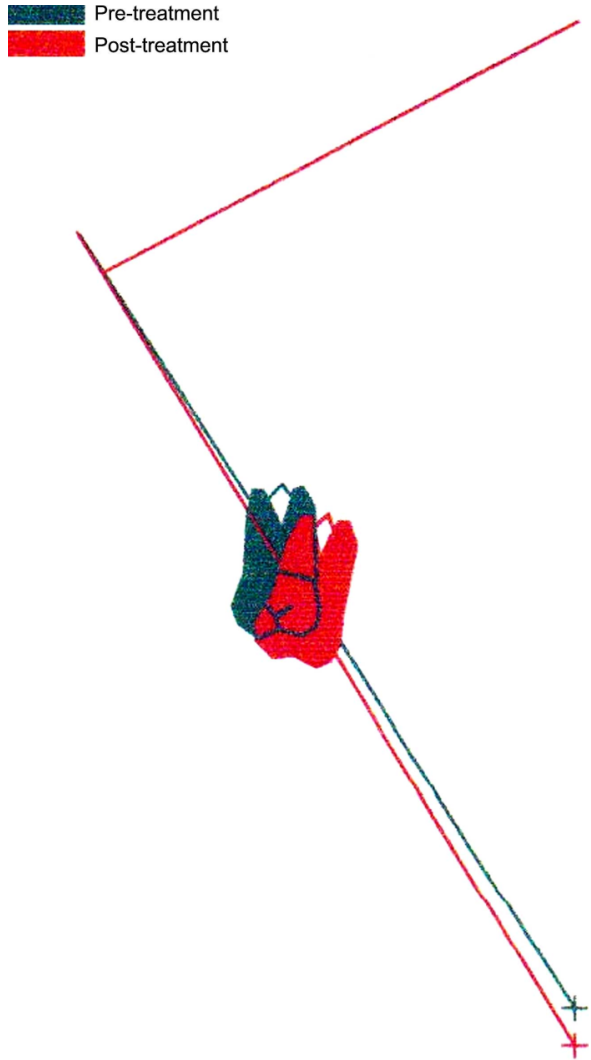

Fig. (2b). Rickett's superimposition along Nasion Basion line at CC and maxillary plane at ANS. Pretreatment in blue and posttreatment in red. The facial axis has opened after treatment.

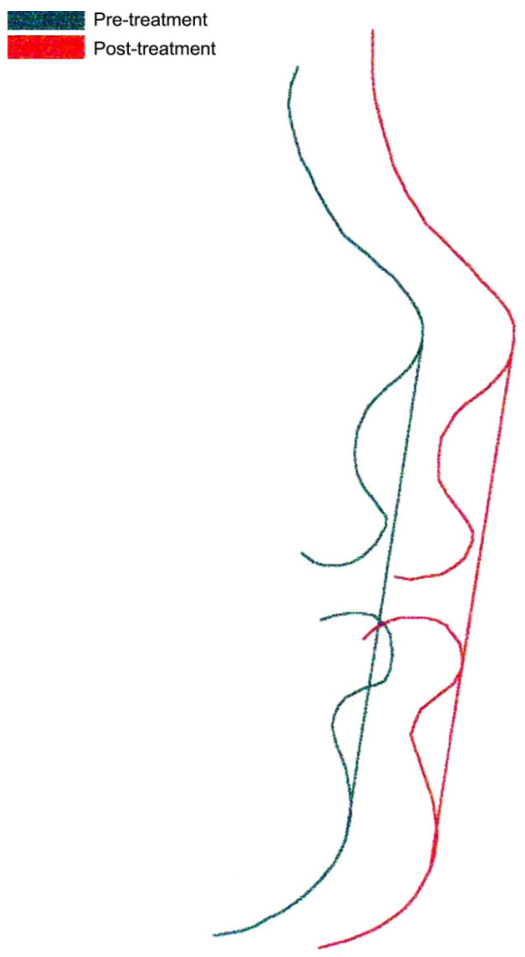

Fig. (2c). Rickett's evaluation of the profile against the E plane. Pretreatment in blue and posttreatment in red. The lower lip in the pre treatment profile was ahead the E plane, the lower lip in the post treatment profile was touching the E plane. 


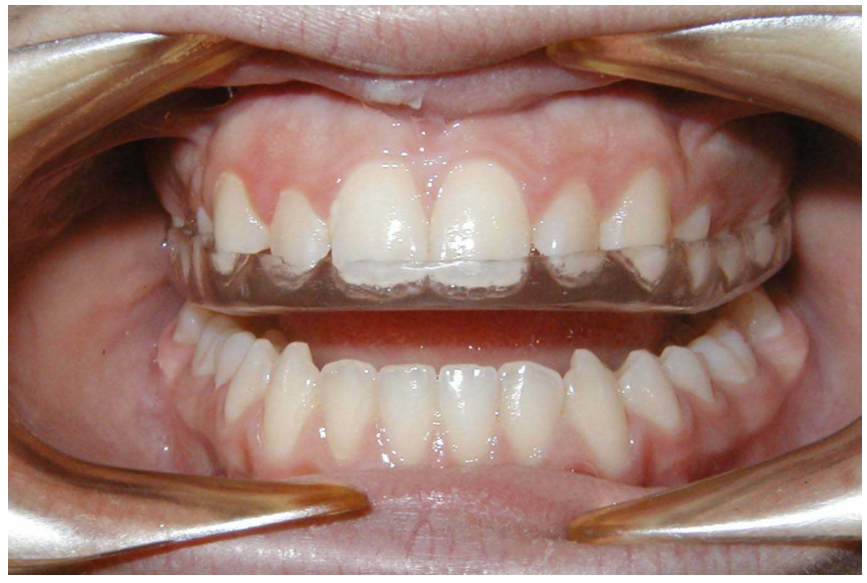

Fig. (3). The splint.

occlusal trauma was bigger in the left side. Radiographs showed that there was severe damage in condyle (Fig. 4). The treatment was orthopedic splint treatment for 1 year, and tongue kinesiology training. As the interdental lingual interposition may produce malocclusion such as open bite, a functional re-education of the tongue can change the lingual position and help to correct the open bite. The design of the splint was similar to that of the first case, but with several

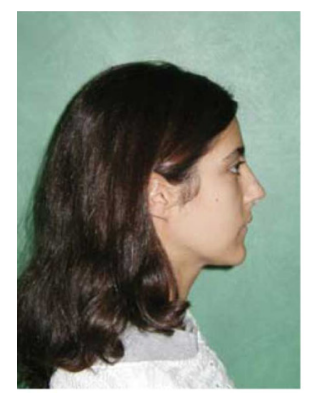

a

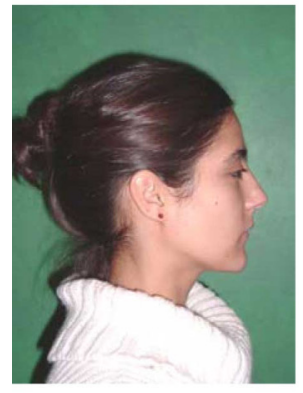

e

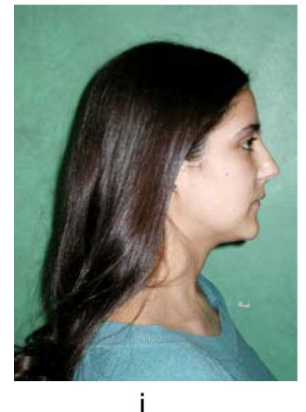

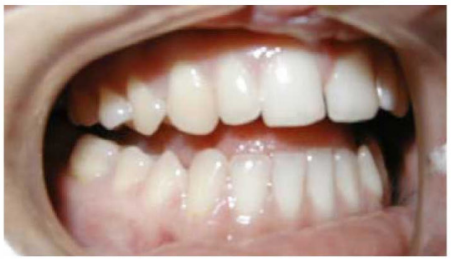

b

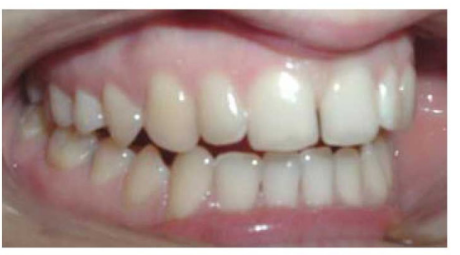

f

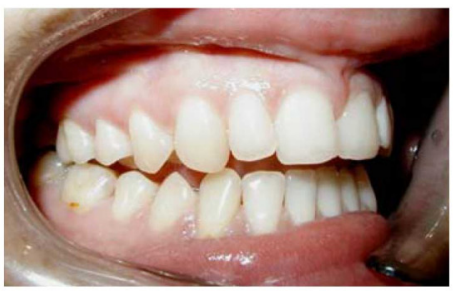

j contacts points in the posterior and medial regions to retract the condyle away from the fossa. We use a bigger splint (3 $\mathrm{mm}$ ), in the posterior and medial sector, to increase the vertical dimension in the mandible position, to protect the TMJ from further injury. The patient used the splint during bedtime and also during daytime for some hours at the beginning of the treatment period when she was still painful. The patient was seen once a week during this stage of treatment until the pain disappeared, subsequent appointments were spaced at 2 weeks interval. The treatment strategy was to let the TMJ to heal by increasing the posterior height so as to produce an anterior rotation and to close the anterior open bite. There was condyle and mandibular growth and gradual improvement in anterior open bite as shown in the radiographs and in the superimpositions of the pre- and postoperative radiographs (Figs. 4-6).

\section{DISCUSSIONS}

The technique illustrated in this article utilized the adaptive capacity of TMJ tissues in the treatment of TMD. At early as 1975, James McNamara [7] pointed the functional adaptations in the TMJ. In animal studies [8-10], forward positioning of condyles increased condylar growth and remodeling of the glenoid fossa in both growing and mature animals. This adaptive capacity of TMJ tissues has also been

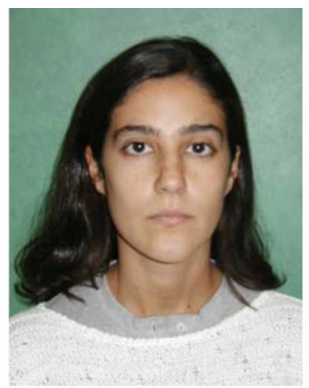

C

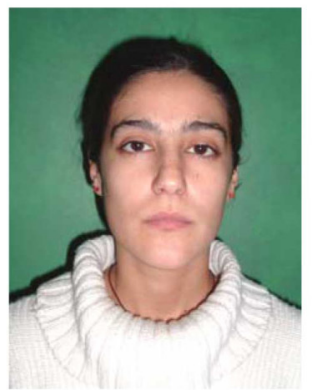

g

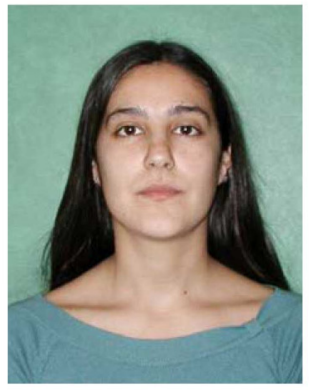

k

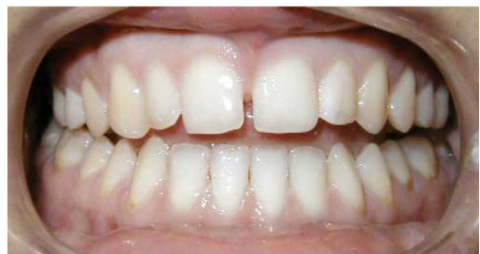

d

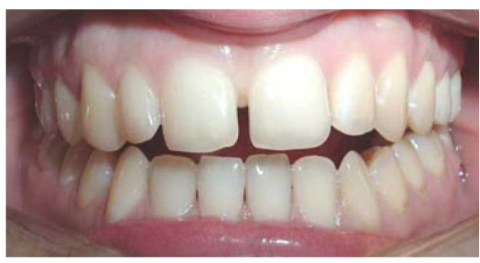

$\mathrm{h}$

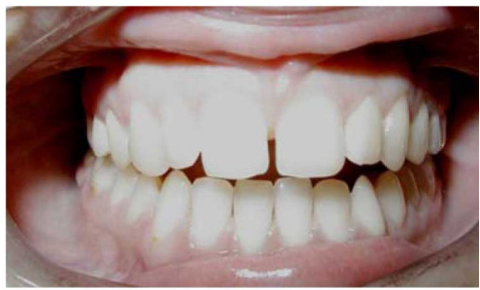

Fig. (4). Case 2 cephalometric radiographs. 


\section{Left TMJ \\ Initial and Control Tomography}

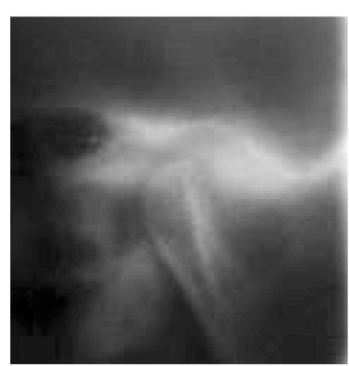

a

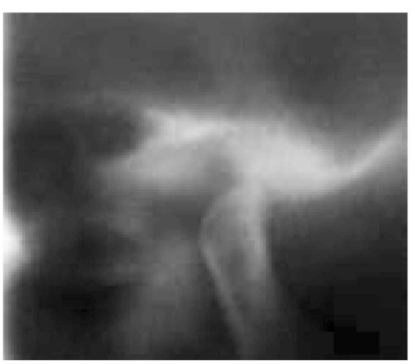

b

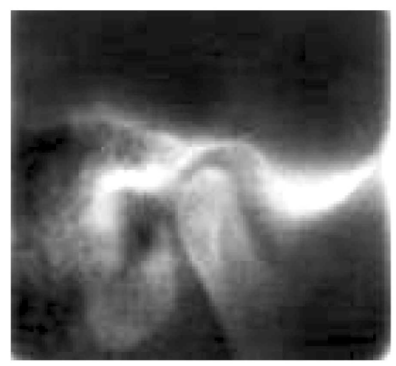

C

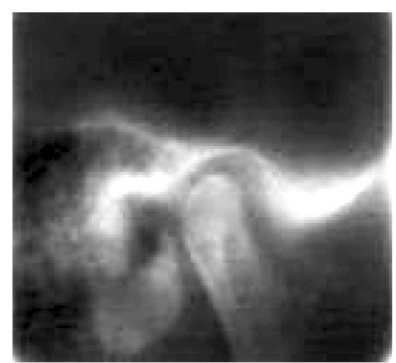

d

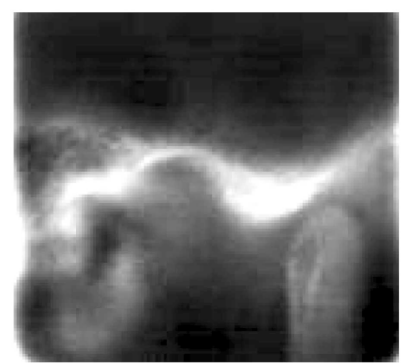

e

\section{Right TMJ \\ Initial and Control Tomography}

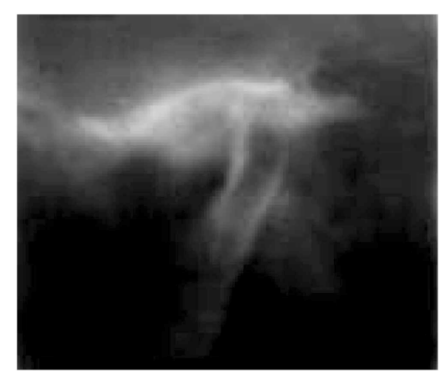

f

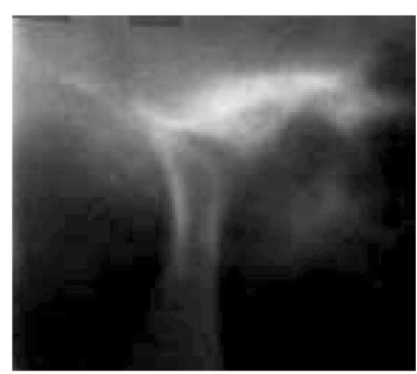

g

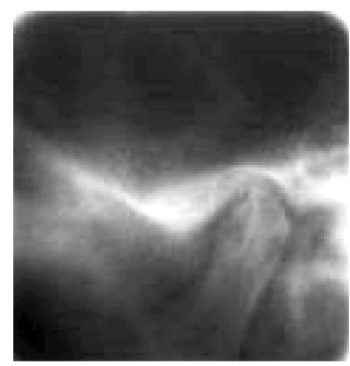

$\mathrm{h}$

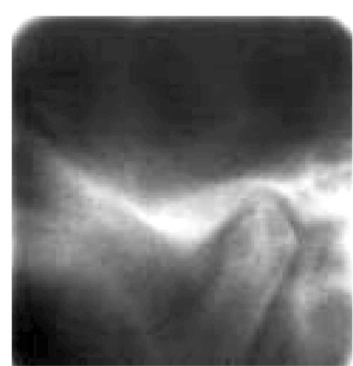

i

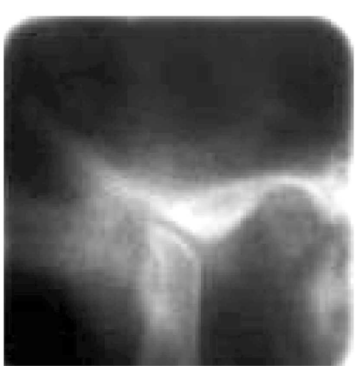

j

Fig. (5). Case 2 TMJ radiographs.

demonstrated in clinical studies in occlusal equilibration [11].

The biological basis of the TMJ remodeling has been investigated. The ground substance /extracellular matrix composition of the articular surfaces of the TMJ is involved in growth and remodeling process of the TMJ. Proteoglycans in the extracellular matrix provide resilience and resistance to compression to articular tissues, examples of these include 


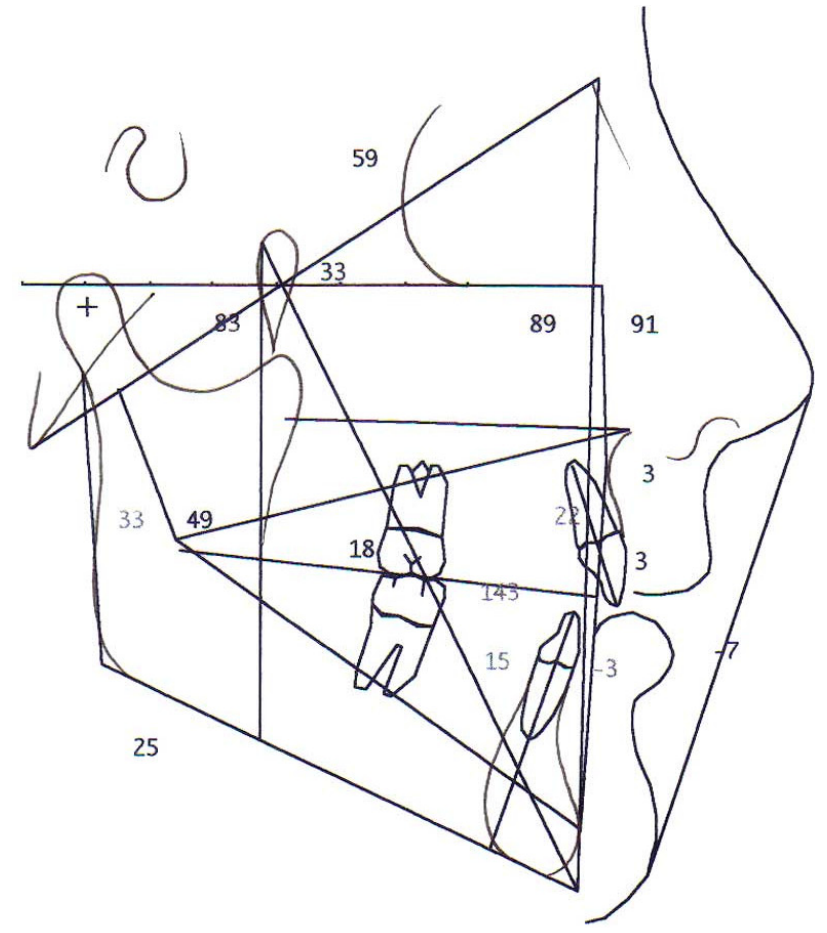

a

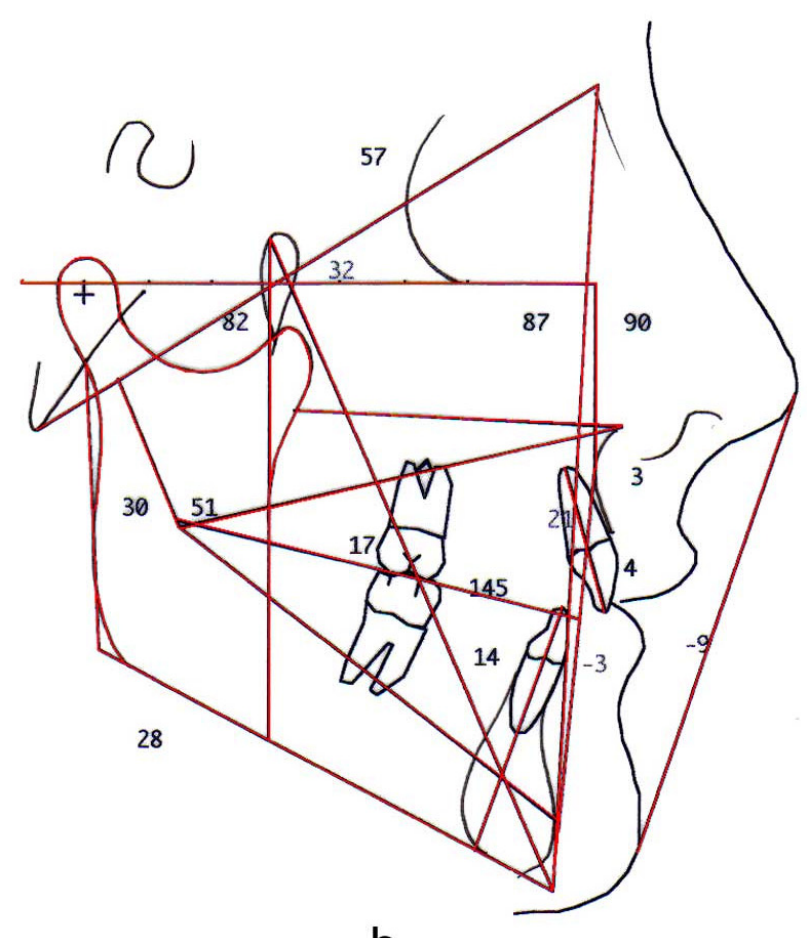

b

Fig. (6). Case 2 Rickett's analysis. Pretreatment in blue and posttreatment in red. By comparing the relative relation between the condyle and the Frankfurt plane before and after treatment, condyle growth can be detected.

versican and aggrecan and they are large proteoglycans found in the extracellular matrix of the mandibular condyle. Aggrecan is mainly responsible for shock absorption and versican and its homologues may be involved in the control of cell proliferation and differentiation [12]. Thus the matrix components of the mandibular condyle seem to be adapted to its special functional needs.

Mesenchymal cells in the deeper lawyer of fibrous connective tissue that covered the articular bone surface in the TMJ condyle, eminence and fossa, can differentiate into fibroblast, chondroblast and osteoblast during articular tissue healing. Change in compressive forces will create changes in extracellular matrix proteoglycans and mesenchymal cells activities. In the TMJ development period mesenchymal cells distribution are very important for TMJ growth and future shape [13].

Take unilateral raising the occlusion by an oral appliance, such as a biteplate, as an example. Unilateral bite raising in a rat model appeared to have induced an increase in the expression of aggrecan in the condylar cartilage and a versicanrelated proteoglycan in the TMJ disc and the articular surface of the condyle [13]. This was suggested to be caused by an increase in the magnitude of compressive forces in the TMJ during unilateral bite raising [14].

In the appliance described in this article, such as in case 1 , the contact of the posterior zone of the splint increased the posterior vertical dimension and unloaded the TMJ tissues and these may in turn induce changes in the expressions of versican, aggrecan and other proteoglycans in the extracellular matrix of the condyle, fossa and articular eminence causing adaptive remodeling of the TMJ. After TMJ remodeling the mandibular position may position either forward and downward or backward and downward depending on clinical situations. From the clinical point of view we observed that this splint decreased the compressive forces against the cranial base, resulting in a decrease in the magnitude of headache, cervical related problems and ear pain.

It appears that any factor that contributes to a change in the biomechanics of the masticatory system can provoke a change in the structure of the TMJ. This includes change in occlusion, loss of teeth, orthodontic manipulation and excessive parafunctional habits. The effects of mechanical compression or tension to the TMJ are influenced by their magnitudes, durations and the adaptive capacity of the host. Temporomandibular joint compression is the most common cause of the structural change of the TMJ. TMJ remodeling in response to the correction of occlusal and/or skeletal malrelationships. Occlusal therapy can produce joint compression by changing the condylar position during or after treatment. Compression results in some degree of resorption of the condyle [15].

In case 2 , idiopathic condylar resorption and progressive condylar resorption is a specific condition that affects the TMJs and most commonly occurs in teenage girls [16]. It causes mandibular condylar resorption and, with shortening of the condyle, it can create occlusal (jaw relationship) and musculoskeletal instability, resulting in the dentofacial deformities, TMJ dysfunction and pain. The recommended treatment for this pathology included splint therapy to minimize joint loading, non-loading orthodontic therapy and orthognathic surgical procedures after the disease had been subsided for 6 to 12 month. These two cases demonstrated an alternative treatment modality with splint is possible. Fur- 
ther studies are needed to substantiate its effectiveness and to compare this with other treatment modality.

\section{CONCLUSION}

It is possible for orthopedic splint with medial and posterior contacts only to produce TMJ remodeling in young and adult patients and to improve the TMD. Further clinical studies are warranted.

\section{REFERENCES}

[1] Carlsson GE, LeResche L. Epidemiology of temporomandibular disorders. In: Sessle BJ, Bryant PS, Dionne RA, Eds. Temporomandibular disorders and related pain conditions. Seattle, WA: IASP Press; 1995; pp. 211-26.

[2] Dworkin SF, Huggins KH, LeResche L, et al. Epidemiology of signs and symptoms in temporomandibular disorders: clinical signs in cases and controls. J Am Dent Assoc 1990; 120: 273-81.

[3] Von Korff M, Dworkin SF, LeResche L, Kruger A. An epidemiologic comparison of pain complaints. Pain 1988; 32: 173-83.

[4] Rugh JD, Solberg WK. Oral health status in the United States: temporomandibular disorders. J Dent Educ 1985; 49: 398-406.

[5] Gray RJM, Davies RJM, Quayle AA. Temporomandibular disorders: a clinical approach. B.D.A.; London: 1995.

[6] McNeill C. Temporomandibular disorders. Guidelines for classification assessment and management. Quintessence; Illinois: 1993.

[7] McNamara JA. Functional adaptations in the temporomandibular joint. Dent Clin North Am 1975; 19: 457-71.
[8] Hinton RJ, Carlson DS. Histological changes in the articular eminence and mandibular fossa during growth of the rhesus monkey (Macaca mulatta). Am J Anat 1983; 166: 99-116.

[9] Rabie ABM, Xiong H, Hagg U. Forward mandibular positioning enhances condylar adaptation in adult rats. Eur J Orthod 2004; 26: 353-8.

[10] Rabie ABM, She TT, Harley VR. Forward mandibular positioning up-regulates SOX9 and type II collagen expression in the glenoid fossa. J Dent Res 2003; 82: 725-30.

[11] Carlsson G, Oberg T. Remodeling of the temporomandibular joint. In: Zarb G, Carlsson G, Eds. Temporomandibular joint function and dysfunction. Mosby; St. Louis, MO: 1979. pp. 155-174.

[12] Roth S, Muller K, Fischer DC, Dannhauer KH. Specific properties of the extracellular chondroitin sulphate proteoglycans in the mandibular condylar growth centre in pigs. Arch Oral Biol 1997; 42: 63-76.

[13] Wurgaft R, Montenegro MA. Remodelacion Articular. In: Desarrollo y estructura de la articulacion temporomandibular. Servimpres; Santiago: 2003; pp. 165-90.

[14] Mao JJ, Rahemtulla F, Scout PG. Proteoglycan expression in the rat temporomandibular joint in response to unilateral bite raise. J Dent Res 1998; 77: 1520-8.

[15] Arnett GW. Progressive mandibular retrusion-idiopathic condylar resorption. Part II. Am J Orthod Dentofac Orthoped 1996; 110 (2): 117- 27.

[16] Wolford LM. Idiopathic condylar resorption of the temporomandibular joint in teenage girls (cheerleaders syndrome). BUMC Proc $2001 ; 14$ (3): 246-52. 